\title{
Die Stellung halten. Marktstrategien und Positionskämpfe in Berlins Internetbranche
}

\author{
Von Alexandra Manske
}

Zusammenfassung: Der Beitrag beleuchtet Marktstrategien und Positionskämpfe von Webdesignern in Berlins Internetbranche. Empirische Basis ist eine qualitative Feldstudie, die auf Intensivinterviews mit hochqualifizierten, freiberuflichen Webdesignern sowie auf Feldbeobachtungen beruht. Die Strukturbeschreibungen des Erwerbsfeldes werden mit der Fragestellung verknüpft, in welcher Weise sich das Ende des »Dot.Com-Booms « im Jahr 2001 auf die Logik des Feldes wie z.B. auf Interaktions- und Konkurrenzbeziehungen auswirkt und welche Strategien die Akteure entwickeln, um sich unter den verschärften Konkurrenzbedingungen in der Internetbranche sozial zu positionieren. These ist, dass die Soziallage von Webdesignern ein spezifisches Unsicherheitspotenzial enthält, das sich im Verlauf des branchenspezifischen Strukturwandels zu einer prekären sozialen Lage auswächst. Anhand einer Typologie dreier verschiedener, subjektiver Marktstrategien (»Unternehmer «, »Dienstleister «, »Künstler«) wird vor dem Hintergrund der skizzierten Entwicklung dargelegt, auf welche Weise die Akteure auf die Feldkontraktion antworten. Als Ergebnis ist zum einen festzuhalten, dass sich die befragten Webdesigner aufgrund ihrer hohen Ressourcenausstattung in einem Zwischenraum von Privilegierung und Prekarisierung befinden. Zum anderen zeigt sich, dass sich dieser sozialstrukturelle Zwischenraum mit der Feldkontraktion zu einer typologisch differenzierten Prekarisierung auf hohem Niveau verengt und insofern Rückschlüsse auf den Strukturwandel von Arbeit zulässt.

Ich glaub, so die Zeiten, dass man 'ne Ausbildung macht und denn bis ins Rentenalter am selben Bankschalter oder in derselben Fleischerei hockt, das ist sowieso gegessen. Tja, dann macht man halt 5, 6 andere Sachen. Ich glaube, das ist eh so der Weg, wo es hingehen wird.

Mit diesen Worten formuliert die 32-jährige Webdesignerin Sara ihr Lebensgefühl und beschreibt die mittlerweile konsensfähige Einschätzung, dass sich das deutsche Sozialmodell überlebt hat (Kaufmann 2003). Vorbei seien die Zeiten, dass man auf traditionelle soziale Sicherheiten wie einen linearen und sozial abgesicherten Erwerbsverlauf setzen könne. Angesagt sei statt dessen ein offensiver und flexibler Umgang mit unsteter werdenden und kontingenten Lebens- und Berufsverläufen.

Sara ist diplomierte Architektin. Doch schon während des Studiums hegte sie den Verdacht, dass sie trotz eines »akademischen Studiums keine glanzvolle berufliche Karriere « (Müller 1998, S. 97) erreichen würde - und dieser Verdacht verfestigte sich in ihrer beruflichen Orientierungsphase. Anstatt jedoch »blauäugig«, so die Architektin, an zertifizierten Qualifikationen festzuhalten, nach einer herkömmlich gesicherten, sozialen Position und nach einer »festen Stelle« zu suchen, stieg sie im Jahr 1999 als Free Lancer in die boomende New Economy ein. Seitdem versucht sie, ihre Existenz als solo-selbständige Internet-Dienstleisterin zu bestreiten. Neben dem, dass ihr »das mit dem Web « $\gg$ Spaß \&emacht hat, findet sie es auch von Vorteil, »auf niemanden angewiesen « zu sein. Sie wollte »mitmischen« in der Internetbranche - also in einem Feld jenseits wohlfahrtsstaatlicher Arbeitsmarktprozesse sowie standardisierter Erwerbsstrukturen, kurz, in einem Feld, dessen soziale Strukturen radikal marktvermittelt sind.

Was es heißt, unter radikal marktvermittelten Bedingungen zu agieren und den Lebensunterhalt sowie den Erwerbsalltag abseits wohlfahrtsstaatlicher Bezugssysteme zu gestalten, wird im Folgenden erläutert. Die Fallgeschichten erzählen von einer Verunsicherung von Lebenslagen, die nicht mehr nur den gesellschaftlichen Rand, sondern zunehmend auch die

Soziale Welt 57 (2006), S. $157-175$ 
gut qualifizierte, soziale Mitte erfasst (vgl. Vester et al. 2001). Empirische Basis der Ausführungen ist eine qualitative Feldstudie in Berlins Internetbranche. Die Befunde stützen sich auf 19 leitfadenorientierte, teilbiografische Interviews sowie auf Feldbeobachtungen. Die Erhebung des zentralen Datenmaterials erfolgte zwischen Sommer 2001 und Frühjahr 2002. ${ }^{1}$ Die Befragten haben teils einen Hochschul- oder Fachhochschulabschluss, teils sind sie Studienabbrecher; einige absolvierten eine vom Arbeitsamt finanzierte Weiterbildung zum »Screendesigner «. Ihre Berufserfahrungen sind unterschiedlich und reichen vom Berufseinstieg bis zu zehn Jahren Erwerbserfahrung. Die Geburtsjahrgänge liegen zwischen 1964 und 1975. Gut die Hälfte der Interviewten lebt in einer festen, aber überwiegend kinderlosen Beziehung. Ihr Verdienst beträgt $10.000-30.000$ Euro/Jahr.

Die Befunde belegen, dass es den Befragten um nichts Geringeres als um soziale »Deklassierungskämpfe « (Bourdieu 1987) geht, darum also, ihre soziale Stellung zu wahren oder zu verbessern, indem sie sich »noch schwach bürokratisierten Sektoren des sozialen Raums zuwenden« (Bourdieu 1987, S. 249). Zugleich handelt es sich nicht um passive Abwehrstrategien und um die altbekannte Frage, ob sie die Not in eine radikal marktvermittelte Branche getrieben hat (vgl. Bögenhold/Leicht 2000). Vielmehr erhoffen sich die Akteure von einer marktvermittelten sozialen Lage sowohl einen höheren Grad an Selbstbestimmung als auch eine eigenverantwortliche Kompensation gesellschaftlicher Instabilitäten. Dafür nehmen sie eine potenziell prekäre Lebenslage in Kauf. Die Befragten ziehen es also vor, sich direkt am Markt zu behaupten, als sich in aus ihrer Sicht gleichfalls unsichere, abhängig institutionalisierte Beschäftigungsverhältnisse zu begeben. Lässt sich dieser Prozess allgemein als Individualisierungsphänomen lesen, indiziert er im besonderen eine Soziallage, die auf das Prekarisierungspotenzial marktvermittelter Lebenslagen hinweist. Eben diesen Blickwinkel werde ich nachfolgend einnehmen und entlang der empirischen Untersuchungsbefunde diskutieren.

So werde ich (1) die Internetbranche in ihren strukturellen Eigenheiten darstellen und schildern, wie sich das Feld nach dem Ende des New-Economy Booms entwickelt hat. Hier sollen sowohl allgemeine Einblicke in die Internetbranche gewährt werden (1.1-1.3) als auch feldspezifische Konkurrenzstrukturen in ihrem Wandel geschildert werden $(1.4 ; 1.5)$. In einem Zwischenfazit werde ich den branchenspezifischen Strukturwandel als marktgetriebene Bürokratisierung charakterisieren sowie anhand verschiedener Dimensionen darlegen, inwieweit er ein Prekarisierungspotenzial für die Soziallage der Akteure impliziert (2). Vor diesem Hintergrund erläutere ich sodann unterschiedliche Marktstrategien, mittels derer sich die Befragten im Feld positionieren (3). Abschließend werden die empirischen Befunde resümiert sowie die Konflikte zwischen den Anforderungen des zu erläuternden Erwerbsmodells und den Problemen seiner Realisierung zusammengefasst (4).

\section{Marktplatz Internetbranche}

Die Internetbranche ist nach wie vor unterbestimmt und wenig erforscht. Zunächst galt sie als Paradebeispiel wissensintensiver Dienstleistungen sowie als ein moderner und unkonventioneller Erwerbsmarkt, in dem sich alles um digitale Dienstleistungen dreht (Batt et al. 2001). Sie entwickelt sich seit Anfang der 90er Jahre als Bestandteil der New Economy ${ }^{2}$, baut auf der Grafik- und Werbebranche sowie auf der IT-Branche auf und ist daher eine »Hybridbranche« (Wolf 2004, S. 2) im Schnittfeld klassischer und neuer Mediensektoren

1) Die Erhebung und Auswertung der Interviews folgt den verstehenden Grundlagen einer Theorie sozialer Praxis (vgl. Bourdieu et al. 2005). Die einzelnen Auswertungsschritte des Datenmaterials erfolgte in technischer Anlehnung an die Grounded Theory als »methodische Leitlinie, die jeweils modifiziert werden muss.«(Strauss 1998: 33) Die Modifikation bestand darin, dass in einem fortgeschrittenen Stadium der Fallanalysen das streng induktive Vorgehen mit einer Feldanalyse verknüpft wurde. 
(Christopherson 2001). Webdesign selbst meint die technische Erstellung und grafische Gestaltung produktionsbezogener sowie konsumorientierter Websites und ist seit 1990 möglich; seitdem existiert das World Wide Web (WWW), ein Zusammenschluss von Servern im Internet (Kotamraju 2002). ${ }^{3}$

Strukturell den Künstlerarbeitsmärkten ähnelnd, koexistieren hier wie da selbständige und abhängige Beschäftigung bei einem hohen Selbständigen-Anteil. Die Zugangswege sind nicht professionalisiert oder institutionell eingehegt, Berufsbezeichnungen infolgedessen nicht geschützt. Die Beschäftigungssicherheit ist gering, das Bildungsniveau ist überdurchschnittlich, doch das Einkommen tendenziell unterdurchschnittlich hoch; schließlich sind sowohl der Künstlerarbeitsmarkt als auch die Internetbranche ein großstädtisches Phänomen (Haak/Schmid 2001; Henninger 2004).

Doch während sich Kulturschaffende über die kostengünstige Künstlersozialkasse (KSK) sozial versichern können (Gottschall/Schnell 2000), bleiben Webdesigner bei der KSK auBen vor. Die Befragten müssen sich deshalb entweder in die KSK, wie sie sagen, »reintricksen «, sich in einen Rechtsstreit begeben oder auf sozialstaatliche Absicherung verzichten. ${ }^{4}$ Insofern ist die soziale Lage von Webdesignern in vergleichsweise schärferer Weise marktbestimmt.

Zudem setzte nach dem Zusammenbruch der technologieorientierten Aktienmärkte (Nasdaq und NEMAX-Index) im Jahr 2001 eine rasante Kontraktion des Feldes ein, während der die Branche nicht nur ihren Glamour einbüßte, sondern auch einen Großteil ihrer Beschäftigungsverhältnisse (Mayer-Ahuja/Wolf 2004). So ist das herausragende Moment der Internetbranche der zeitlich äußerst kurze Zyklus von einem expandierenden zu einem schrumpfenden Erwerbssegment.

Der Einbruch und der sich anschließende Strukturwandel hat allerdings dazu beigetragen, dass sich eine eigenständige und unterscheidbare Multimediabranche heraus gebildet hat. Diese Entwicklung wird nachfolgend geschildert.

\subsection{Vom »Boom« zum »Bust «}

Als Schaumkrone der New Economy wurden die ersten Multimedia-Agenturen, die »DotComs « bzw. »Startups « in den beginnenden 90er Jahren in New York City und San Francisco gegründet (Kotamraju 2002). Nach Deutschland gelangte der Begriff »New Economy« einige Jahre später und war zwischen etwa 1998 und 2001 ein medialer Dauerbrenner. Hierzulande konzentrierte sich die New Economy auf Großstädte wie Berlin, Köln, Hamburg und München. In Berlin bündelten sich die Startups im Bezirk Mitte auf wenige Straßenzüge

2) Der Begriff »New Economy« ist ein verbaler Platzhalter für die Entstehung und Ausbreitung technologieorientierter (Klein)Unternehmen, die vom anhaltenden Wirtschaftswachstum profitierten und auf einer digitalisierten Produktion von Dienstleistungen aufbauen (Ortlieb/Rokitte 2004).

3) Die Ursprünge der digitalen Datenübermittlung (ARPAnet) liegen in den 60er Jahren des amerikanischen Verteidigungsministeriums und waren Teil der militärischen Sicherheitsstrategie im Kalten Krieg. In den 70er Jahren wurde ein weltweites Kommunikationsnetz entwickelt, das die Kommunikation von Computern erlaubt und auf host-to-host (TCP) und internetworks protocol (IP) basiert. Zivil nutzbar wurde das Internet 1983, als ARPAnet in eine zivile und eine militärische Komponente (MILnet) aufgeteilt wurde. 1983 wurde auch das Domain-Name-System entwickelt. Seitdem gibt es Internetadressen mit der Endung .com und .org. 1989 wurde am europäischen Kernforschungszentrum CERN die Programmiersprache HTML (Hypertext Markup Language) entwickelt und 1993 der erste Browser. Bis 1995 wurde das die Vernetzung koordinierende Netzwerk des Internet von der US-Regierung betrieben und im selben Jahr privatisiert (Castells 2001; Meschnig/Stuhr 2001).

4) Allerdings sind beim Bundessozialgericht mehrere Einzelklagen anhängig, die u.U. zur sozialrechtlichen Anerkennung von Webdesignern führen werden. 
und wuchsen dort bis Ende der 90er Jahre auf eine Anzahl von ca. 400 an (Stuhr 2003, S. 167). Angelehnt an den New Yorker Ausdruck Silicon Alley - seinerseits eine Abwandlung des kalifornischen Silicon Valley - kursierte für das Zentrum der Berliner Internetbranche in der Chausseestraße die Bezeichnung »Little Silicon Alley« sowie »Silicon City«. »Dort zu arbeiten«, kommentiert Mathias Stuhr (2003, S. 167), »war nicht nur richtig hip, sondern »irgendwie« gar kein richtiges Arbeiten mehr«. Ob »richtiges Arbeiten« oder nicht - ein Moment, aus dem die New Economy ihre Anziehungskraft bezog, war die Tatsache, dass es sich um ein unscharf umrissenes und gestaltungsoffenes Feld handelte, in dem es während des Booms nicht zuletzt möglich war, »mit wenig Arbeit am Bildschirm ganz viel Geld zu verdienen.« (Meschnig/Stuhr 2001, S. 29)

Im Jahr 2002 indes war Berlins »Silicon City« bereits Vergangenheit und die »Startups« aus der Chausseestraße mehrheitlich wieder von der Bildfläche verschwunden. Denn mit dem Ende der New Economy-Euphorie fanden auch die Vorstellungen vom schnellen Geld am Bildschirm ein jähes Ende (Neff 2002).

So setzte nach 2001 eine rapide Schrumpfungsphase ein. ${ }^{5}$ Doch wegen der weitgehend informellen Branchenstrukturen lässt sich die Anzahl und Form der Beschäftigungsverhältnisse nicht genau beziffern. Während die Angaben über die Gesamtbeschäftigungszahlen für das Jahr 2001 zwischen 72.000 und 151.000 schwanken (Mayer-Ahuja/Wolf 2004), ist insbesondere die Anzahl der Alleinunternehmer/-innen nicht genau bekannt. Verschiedene Schätzungen und einzelne Felduntersuchungen gehen aber davon aus, dass deren absolute Zahl im Jahr 2001 bei etwa 60.000 lag (Rehberg et al. 2002; Vanselow 2002). Die Dominanz von Klein- und Kleinstunternehmen in der Internetbranche entspricht damit dem allgemeinen Trend vom Arbeitgeber- zum Solo-Selbständigen (Leicht/Philipp 2005).

Die Expansionsphase der Internetbranche war also auf ein Zeitfenster von drei Jahren beschränkt und steckt entsprechend die erwerbsbezogenen Gelegenheitsstrukturen auf die Phase zwischen 1998 und $2001 \mathrm{ab}$.

\subsection{Kunst oder Technik?}

Webdesign war anfangs eine relativ undifferenzierte Tätigkeit. Bis Ende der neunziger Jahre zeichneten sich die Tätigkeitsprofile durch »flexible skills with fluid titles « (Kotamraju 2002, S. 6) aus. Neben allgemeinen Sprachfertigkeiten, dem Beherrschen der Programmiersprache HTML sowie dem Wissen darum, wie die jeweilige Software mit verschiedenen Browsern korrespondiert, war anfangs ein nicht näher definierter »Sense of visuality « ausreichend, um in der Internetbranche Fuß zu fassen. ${ }^{6}$ Ausgefeilte Technikkenntnisse waren in der Anfangsphase nicht notwendig. Die offene Feldstruktur begünstigte, dass sowohl Kunstinteressierte mit einem Interesse an der Verschaltung alter und neuer Medien sowie z.B. Informatiker in das Feld einmündeten.

Zügig jedoch polarisierte sich Webdesign in »Art vs. Code« (Kotamraju 2002, S. 9). Auch wenn die Polarisierung von Programmierern einerseits und Designern andererseits in den USA ausgeprägter war, war sie auch hierzulande ein Distinktionsmodus (Meschnig/Stuhr

5) Pixelpark ist ein prominentes Beispiel für das Ende des New Economy Booms. 1995 in Berlin als eine der ersten Multimediaagenturen in Deutschland gegründet, verzeichnete das Unternehmen 1999 mehr als 1000 Mitarbeiter, europaweite Dependancen und 27 Millionen DM Jahresumsatz (Süddeutsche Zeitung 15.12.02). 2002 hatte Pixelpark in Berlin noch 35 Mitarbeiter und wurde 2003 von Bertelsmann aufgekauft.

6) So ein Interviewpartner, den ich 2001 in New York im Rahmen eines Forschungsaufenthaltes traf. Selbiger sowie weitere in New York erhobene Interviews fließen jedoch nicht systematisch in meine Ausführungen ein. 
2001, S. 54). So entstanden Vormachtkämpfe zwischen den unterschiedlichen Gruppen (»Techies« vs. »Designer«). Sie kreisten um die Frage, was Webdesign »wirklich« ist Kunst oder Technik? Durch die rasante, technische Weiterentwicklung (JavaScript, flash etc.) wurde Webdesign alsbald als eine technische Tätigkeit gedeutet, sodass versierte Technikkenntnisse zunehmend über die Marktstellung der Einzelnen entscheiden.

Festzuhalten ist, dass nicht zuletzt wegen der fortschreitenden Kommerzialisierung die »Techies« als Sieger aus den Definitionskämpfen hervorgingen und entsprechende Distinktionsgewinne verbuchen konnten. ${ }^{7}$

\subsection{Formalisierung von Arbeitsstrukturen}

Auf die Spannung von Kunst vs. Technik folgte eine weitergehende Differenzierung des Feldes, welche die Akteure relational zueinander positioniert und mit unterschiedlichen Gestaltungsoptionen für ihren Arbeitsalltag ausstattet. Während es in der Expansionsphase üblich war, Internet-Dienstleistungen aus einer Hand anzubieten, werden nun eine funktionale sowie rationalisierte Spezialisierung im Sinne einer »marktgetriebenen Bürokratisierung « (Wolf 2004, S. 5) zu bis dato unbekannten Erfolgsindikatoren. Diese und weitere »neue « Erfolgsindikatoren führen zu einer horizontalen sowie vertikalen Segmentierung der Tätigkeitsbereiche.

Neben den für Projektarbeit üblichen, kurzfristigen Deadlines zeichnet sich der Arbeitsprozess durch eine flexible Ad-hoc-Abstimmung auf Kundenwünsche aus: alles schnell und am besten schon gestern erledigen (Mayer-Ahuja/Wolf 2004, S. 19ff.). Die Aufgabenkomplexe umfassen drei systematische Bereiche und Ebenen: (1) Content (inhaltliche und grafische Konzeption sowie Design), (2) Programmierung, (3) Management. (4) ließe sich die Akquise, d.h. der Kundenkontakt hinzu fügen (Batt et.al. 2001, S. 11). Die Definitionsphase im Bereich Content beginnt mit dem Kundenkontakt, während dem das finanzielle und inhaltliche Auftragsvolumen verhandelt wird. Daran schließt sich die Konzeptionsphase an: das »Story-Board « wird entwickelt und dem Kunden präsentiert. Nach der dann erfolgenden Feinkonzeption und grafischen Erstellung eines Offline-Prototyps der Webseite wird mit der Implementation begonnen. Dazu gehört die Anwendungsprogrammierung, die Abstimmung mit den jeweiligen Browsern, die Dokumentation des Quellcodes etc. In dieser Phase sind in der Regel Rücksprachen mit den Kunden notwendig, da Offline-Prototyp und Online-Anwendung (das sogenannte »Front End«) wegen mangelnder technischer Übereinstimmung häufig voneinander abweichen.

Hinzu kommt, dass den Auftraggebern mehrheitlich das Wissen um die technische Machbarkeit fehlt, sodass sich die Kundenarbeit nicht auf den Produktionsprozess der Webseite beschränkt, sondern auch eine post-produktive Seiten- und Kundenpflege umfasst, z.B. wenn nach der Präsentation des Offline-Prototyps »der Kunde jetzt plötzlich alles rot anstatt blau haben will« $\left(030^{8}\right.$; vgl. auch Perrons 2003).

Im Verlauf der Feldkontraktion schrumpfen also nicht nur die Aufträge und das Honorar, sondern es formalisieren sich auch die Arbeitsstrukturen hin zu einer modernisiert tayloristischen Organisationsweise.

7) Die Definitionskämpfe spiegeln sich in den Interviews deutlich wider: »Die Leute, die meinen, sie können »web-designen«, [...] die also nur mit Editoren (Editoren sind vorgefertigte Programme wie z.B. Photoshop und erfordern keine Programmierkenntnisse, AM) umgehen können und das war's, die haben bald keine Chance mehr.«

8) Die Zahlen hinter den Zitaten geben die laufende Nummer des Interviews an. 
So fechten die Akteure neben den kulturellen Distinktionskämpfen auch funktional-sachliche Kämpfe aus. Vor dem Hintergrund einer geringeren Auftragsdichte etc. führt dies zu einer fraktionsspezifischen Differenzierung im Feld sowie dazu, dass die Radikalität des Marktes mit seinen essenziell ungleichheitsgenerierenden Effekten umso deutlicher hervor tritt. Werfen wir nun einen Blick auf die Konkurrenzstrukturen.

\subsection{Direkte und indirekte Konkurrenz}

Wie konstituieren sich Interaktions- und Konkurrenzbeziehungen auf dem Marktplatz Internetbranche? Zunächst ist festzustellen, dass der Markt als Schauplatz sozialer Prozesse zugleich ein klassisches Thema der Soziologie ist, wie auch ein lange Zeit brachliegendes Untersuchungsfeld. So stellt etwa Richard Swedberg fest, dass sich die Soziologie nach Webers und Simmels grundlegenden Begriffsklärungen über ein soziologisches Verständnis von »Markt« bis in die mittleren 70er Jahre zu diesem Thema ausschwieg (Swedberg 1994, S. 267). ${ }^{9}$

Insbesondere im deutschen Kontext ist die diesbezügliche, wissenschaftliche Neugierde erst seit kurzem wieder erwacht. Aus verschiedenen soziologischen Perspektiven wird nun eine zeitdiagnostische Bestimmung von »Marktgesellschaften« vorgenommen (z.B. Honneth 2002; Neckel 2001; Rüb 2003). Während es indes Ende der 90er Jahre vorwiegend um eine Analyse von Tauschvorgängen als Strukturierungsprinzip des ökonomischen Systems oder um soziale Voraussetzungen marktförmiger Verfahrensweisen ging (Beckert 1997; Kraemer 1997), wird in jüngerer Zeit ein Wandel von der industriegesellschaftlich geprägten Sozialordnung hin zur Marktgesellschaft identifiziert. Begrifflich von der arbeits- und industriesoziologischen Debatte geprägt, wird von einer Vermarktlichung sozialer Zusammenhänge gesprochen (Sauer/Döhl 1997). Argumentiert wird, dass sich soziale Deutungsmuster wandeln und dem Markt als amorphes Gebilde vielfach eine Selbstevidenz zuerkannt werde, die kaum noch einer normativen Rechtfertigung bedürfe (Neckel et al. 2005; Voswinkel 2000). So definiert etwa Sighard Neckel Marktgesellschaften als »jene institutionellen Formen des modernen Kapitalismus (...), die sich aus der Einbettung des wirtschaftlichen Wettbewerbs in begleitende Sozialnormen (...) sukzessive herauslösen und (...) auf das (...) Prinzip von Angebot und Nachfrage reduzieren.« (Neckel 2001, S. 252)

Obgleich dieser Sachverhalt weitgehend unstrittig ist, lassen sich Sozial- und Konkurrenzbeziehungen mittels ökonomisch fundierter Erklärungsprinzipien nur bedingt einholen, da darin letztlich immer die Annahme mitschwingt, dass es zumindest theoretisch einen idealen Markt geben könnte. Aus ungleichheitssoziologischer Perspektive hingegen stellen sich Wettbewerbsbedingungen immer in Abhängigkeit von der relationalen Stellung im Feld dar, sind also notwendig sozial ungleich (Bourdien 1987), sodass es keinen idealen Markt geben kann. Um daher die prozessualen Aspekte von Konkurrenzbeziehungen heraus zu arbeiten, bietet es sich nach wie vor an, an klassische Erklärungsansätze anzuknüpfen und diese durch Erkenntnisse aus der Netzwerkforschung zu ergänzen.

Danach zeichnen sich marktvermittelte Sozialbeziehungen wesentlich durch zwei Aspekte aus (vgl. Nullmeier 2001; Swedberg 1994). Zum einen durch einen spezifischen Interaktions- sowie zum anderen durch einen spezifischen Konkurrenzmodus. Insbesondere Weber (1922/1972) betont Handlungs- und Interaktionsaspekte, die sich als Preiskampf zwischen

9) Erst in den 80er Jahren erhielt die internationale Debatte durch Marc Granovetter neuen Aufschwung (Granovetter 1985). Der deutschen Debatte attestiert etwa Frank Nullmeier (2001) eine traditionell antithetische Haltung gegenüber einer Analyse des Marktes. Sie fuße auf dem soziologischen »Grundideologem«, dass der Markt gesellschaftlich nicht integrationsfähig sei (Nullmeier 2001, S. 227f). 
zwei Tauschpartnern sowie als Konkurrenzkampf aller gegen aller artikulieren, an dessen Ende eine Austauschbeziehung zwischen einigen Marktteilnehmern steht (Weber 1972, S. 16ff.). Damit, so etwa Nullmeier (2001, S. 229), erhalte Weber Zugang zu Marktprozessen als wettbewerbsorientierte Interaktionsbeziehung bzw. zur sozialen Ordnung von Märkten (vgl. auch Beckert 1997). Wie sich dieser Wettbewerb jedoch als sozialer Prozess konkretisiert, dafür biete Weber keine konzeptionelle Erklärung, weil er sich letztlich auf die sozialen Rahmenbedingungen wie z.B. distinktive Schließungsprozesse konzentriere (Nullmeier 2001, S. 229). Simmel (1903/1983) dagegen fokussiert stärker auf den Wettbewerb als sozialen Prozess. Er differenziert Wettbewerb auf systematischer Ebene und führt zwei Aspekte von Konkurrenz ein. Konkurrenzbeziehungen lassen sich mit Simmel grundlegend als »indirekter Kampf « verstehen. Er tritt in zweierlei Arten auf - als direkter sowie als indirekter Wettbewerb. Zum einen geht es um direkte, rivalisierende Konkurrenz, da beide bzw. beteiligte Parteien auf ein begehrtes Gut zielen. Zum anderen spricht Simmel von einer indirekten Konkurrenz, bei dem sich die Akteure parallel, aber nicht direkt rivalisierend um den Kampfpreis bemühen. Hier »besteht der Kampf überhaupt nur darin, dass jeder der Bewerber für sich auf das Ziel zustrebt, ohne eine Kraft auf den Gegner zu verwenden.« (Simmel 1983, S. 175)

In der Praxis entspinnen sich Positionskämpfe maßgeblich in Netzwerkstrukturen (Jansen 2003). Denn informelle, soziale Netzwerke übernehmen im weitgehend nicht institutionalisierten Erwerbsfeld Internetbranche eine steuerungstheoretische Kompensationsfunktion. Während allerdings in der netzwerktheoretischen Debatte ein ökonomisch rationalisierter Handlungsbegriff dominiert, zeigt sich im Datenmaterial, dass berufliche Netzwerke nicht eindeutig als Resultat kalkulierter Interessen interpretiert werden können. Vielmehr bemühen sich die Akteure unter Einsatz sämtlicher ihnen zur Verfügung stehender Ressourcen um den Aufbau oder die Vertiefung von sozialen Beziehungen, um sich Konkurrenzvorteile zu verschaffen. Generell ist daher festzuhalten, dass Netzwerke von einer Ambivalenz von Konkurrenz und Zusammenschluss (Simmel) geprägt sind, sodass die feldspezifischen Verortungspraxen entlang der individuellen Kapitalausstattung (Bourdieu 1983) einen differenzierten Konkurrenzkampf um berufliche Stellungen sowie um Aufträge widerspiegeln.

Netzwerke entscheiden zudem nicht nur über die Anzahl der Aufträge, sondern auch über ihre Art, z.B. ob eine Website für ein Kulturfestival oder eine Unternehmensberatung erstellt wird. Die Art der Netzwerke und die sie kennzeichnenden Konkurrenzstrukturen sind daher auch nach Volumen und Struktur, d.h. nach der Anzahl der sozialen Beziehungen und der Spezialisierung auf Marktsegmente sowie danach zu unterscheiden, ob die Akteure eher in vertrauensbasierte oder in versachlichte bzw. formalisierte Netzwerke investieren (vgl. Granovetter 1973).

Insgesamt strukturieren sich Konkurrenzbeziehungen in der Internetbranche im Rahmen informeller Netzwerke. Sie stellen ein Geflecht von direkten und indirekten Beziehungen dar. Feldspezifische Netzwerkstrukturen, verstanden als durch soziale Beziehungen konstituierte, prinzipiell hierarchische Gefüge des Konkurrenzkampfs und als Ort, an dem soziales Kapital akquiriert wird, beruhen auf einem hybriden Muster von Konkurrenz und Zusammenschluss. Die Teilhabe an bestimmten Netzwerk-Strukturen entspricht somit nicht nur einer funktionalen Differenzierung des Feldes, sondern stellt notwendig auch eine hierarchische Platzierung im Gefüge diverser branchenspezifischer Netzwerke dar. Sie konturieren sich als horizontales, brückenbildendes Kapital zu Kollegen oder Freunden sowie als vertikal bindendes Sozialkapital zu auftraggebenden Agenturen (Henninger 2004). Kurz, Netzwerke indizieren die relationalen Stellungen der Akteure zueinander. Gestaltung von Marktbeziehungen heißt dann nicht nur, dass den Individuen Marktanforderungen hermetisch entgegen treten, sich gegen die Einzelnen durchsetzen oder von ihnen aufgegriffen werden. In Abhängigkeit von der Stellung in verschiedenen Netzwerken spielen sie sich als verschärfte Kon- 
kurrenzbeziehungen ab. Die feldspezifischen Netzwerkstrukturen sind daher als konkurrenzorientierte Anforderungsstruktur und als ökonomische Durchdringung sozialen Handelns in soziale Beziehungen eingebettet, die nicht nur wirtschaftlichen Rationalitäten folgen (Manske 2005).

\subsection{Veränderte Konkurrenzbeziehungen}

Im Verlauf des branchenspezifischen Strukturwandels verändern sich gleichwohl die Konkurrenzbeziehungen. Während anfangs eine »unglaubliche Offenheit « geherrscht habe und es Aufträge ohne Ende gab, sei da heute »Verschlossenheit, Misstrauen und die Angst «, dass die Konkurrenz schneller ist, so ein Interviewpartner. Denn der Preiskampf mit nicht zuletzt größeren Agenturen zwingt Free Lancer häufig dazu, ihre Dienstleistung nicht kostendeckend anzubieten. Symptome dafür sind etwa häufiges Arbeiten in den Abendstunden oder im Urlaub. Zudem verstärkt sich insbesondere die indirekte Konkurrenz, bei der die Akteure parallel, aber nicht direkt rivalisierend um eine soziale Stellung kämpfen. Symptomatisch für die wachsende indirekte Konkurrenz ist sowohl die bereits erwähnte funktionale Differenzierung als auch die Fokussierung auf bestimmte Marktsegmente wie z.B. die Spezialisierung auf »Industriekunden« oder den Kulturbereich.

Zunächst jedoch beruhten informelle Marktbeziehungen auf sogenannten »Cybersuds« oftmals Produkt des Nachtlebens, für das die New Economy bekannt war. Der »First Tuesday « steht besonders markant für diese Art des Networkings. Zwischen 1998 und 2000 wurde in großstädtischen Zentren der Internetbranche am ersten Dienstag jeden Monats Parties veranstaltet, die als geschäftliche Kontaktbörse dienen sowie die distinktive Aufbruchseuphorie der New Economy symbolisieren sollten. In Berlin fand mithin der per Mundpropaganda in der Szene verbreitete, gewissermaßen als subkulturelle Gegenveranstaltung angelegte »Last Tuesday « mehr Zuspruch.

Dennoch zeichnet sich eine informelle Professionalisierung der Vernetzungsstrukturen ab. Der etwa 2003 national reaktivierte First Tuesday findet in losen Abständen als vergleichsweise formalisierte Podiumsdiskussion statt - unter Beteiligung von z.B. Unternehmensberatungen oder Finanzierungsgesellschaften sowie von ihnen gesponsort. Die informelle Professionalisierung ändert jedoch nichts daran, dass Lobbygruppen bisher keine offizielle Marktmacht haben. So gibt etwa der Deutsche Multimedia Verband (DMMV) Verbandsmitgliedern neben technischen Daten wie Iso-Richtlinien zur standardisierten Produktion von Websites auch »Honorarleitfäden « an die Hand; doch sind diese praktisch nur bedingt relevant, da Honorare individualisiert verhandelt werden und je nach Marktsegment häufig nicht einmal die Hälfte der empfohlenen Honorarhöhe erzielt wird (Vanselow 2002). Demnach entscheidet eher die Struktur als das Volumen des sozialen Kapitals darüber, ob die Tätigkeit in der Internetbranche existenzsichernd ist oder zu einer prekären materiellen Lage führt. Denn von der strukturellen Ausgestaltung der Marktbeziehungen und der Spezialisierung auf Marktsegmente hängt es ab, ob die Aufträge finanziell einträglich sind oder eine prekäre Existenz untermauern.

Ein Weg die verschärften Konkurrenzbedingungen zu handhaben, stellen informelle Zusammenschlüsse dar. Bürogemeinschaften, verstanden als horizontale Netzwerke unter Gleichen (Henninger 2004), sind ein Mittel, der sozial isolierten Tätigkeit als Solo-Selbständige $\mathrm{zu}$ entfliehen und Marktschwankungen »kollektiv« auszugleichen. Hier zeichnet sich ein Spektrum zwischen einer »informellen Verbetrieblichung « und einer »kollektivierten Vereinzelung « ab - damit sind abgestufte Formen inhaltlicher Kooperation gemeint, einmal auf Basis einer Corporate Identity (symbolisiert etwa durch ein gemeinsames Logo), das andere Mal als eher lose inhaltliche Vernetzung im selben Büro. Tatsächlich alleine und Zuhause arbeiten nur wenige Befragte. 


\section{Zwischenfazit: neue Feldbedingungen - anwachsendes Unsicherheitspotenzial}

Zusammenfassend lässt sich der Strukturwandel der Internetbranche zwischen 1998 und 2001 als »marktgetriebene Bürokratisierung ${ }^{10}$ (Wolf 2004) auf den Punkt bringen. In Folge der feldspezifischen Kontraktion formalisieren sich zum einen die Arbeitsstrukturen und verschärfen sich zum zweiten die - direkten sowie indirekten - Konkurrenzstrukturen und Marktbeziehungen. Stichworte sind hier ein steigender Verdrängungswettbewerb, fallende Verdienstmöglichkeiten sowie stärker konturierte Aufgabenkomplexe, die wiederum die Konkurrenzverhältnisse verschärfen.

Zwar erinnert die Diagnose einer marktgetriebenen Bürokratisierung an altbekannte tayloristische Arbeitsstrukturen. Gleichwohl enthüllt der Strukturwandel der Internetbranche hybride Phänomene zwischen informellen und formeller werdenden Feldstrukturen, sodass es sich weniger um eine »nachholende Modernisierung « handelt, als um eine neue Phase radikal marktvermittelter Feldbedingungen.

Die Stellung der Webdesigner hängt nun davon ab, wie sie die Anforderungen einer zunehmend funktionalen Differenzierung der Arbeitsstrukturen aufgreifen, auf welche Marktsegmente sie sich spezialisieren und wie ihr Konkurrenzverhalten aussieht. Nicht zuletzt positionieren sie sich abhängig davon, wie weit sie ihr privates Leben in den beruflichen Dienst nehmen.

Verändert die Feldkontraktion daher zum einen die strukturellen Eigenheiten der Internetbranche, ist mit ihr zum zweiten ein anwachsendes Unsicherheitspotenzial für die eigenverantwortliche Stabilisierung der sozialen Lage verbunden. Strukturell gilt, dass die soziale Lage von Webdesignern aus einer Kollision von neuen Erwerbs- und Arbeitsformen mit industriegesellschaftlichen Ordnungsmustern resultiert. Denn eingebunden in Erwerbsarbeit und sozialpolitisch ausgeschlossen, entspricht ihre soziale Lage einer prekären Inklusion bzw. einem gleichzeitigen Drinnen und Draußen (Simmel). Radikal marktvermittelt war und ist die Lebenslage von Webdesignern also deshalb, weil sie ihre materielle sowie ihre institutionelle Teilhabe ${ }^{11}$ am gesellschaftlichen Leben marktabhängig erwirtschaften und ihre Lebensführung in synchroner sowie diachroner Hinsicht »marktgerecht« gestalten müssen. $\mathrm{Ob}$ und in welchem Ausmaß sich Webdesigner gegen allgemeine Lebensrisiken absichern können, hängt entsprechend ihres Erwerbsstatus' ausschließlich von der Auftragslage ab.

Zur Hochzeit der New Economy schien diese Soziallage sowie die damit verknüpfte Deprivierung sozialer Rechte (Marshall 1992) noch ein halbwegs kalkulierbares Risiko zu sein - oder zumindest eines, das sich ignorieren ließ. Im Verlauf der Feldkontraktion kristallisiert sich jedoch heraus, dass »radikal marktvermittelt« in vielen Fällen synonym ist mit »prekär«.

So führten die Interviewten vielfach an, dass sie ohne gelegentliche Finanzspritzen ihrer Anverwandten nicht über die Runden kommen würden. Zwar reicht ihr Einkommen in der

10) Hier wird »marktgetriebene Bürokratisierung « als Schlüsselbegriff für eine neue, zugespitzte Phase radikal marktvermittelter Bedingungen genutzt. Damit möchte ich insbesondere die Bedingungen der feldspezifischen Kontraktionsphase und somit die zeitliche Ebene akzentuieren. Demgegenüber spricht Harald Wolf, dem ich den Begriff entlehnt habe, von einem gegenseitigen Steigerungsverhältnis: »Je unmittelbarer die Abhängigkeit vom Markt, desto stärker die Tendenz interner Strukturierung und Hierarchisierung « (Wolf 2004, S. 5)

11) Gesellschaftliche Teilhabe lässt sich mit Kronauer (2002) als ein mehrdimensionaler Prozess und Zustand verstehen. Sie strukturiert sich über die Partizipation an Erwerbsarbeit, über die formale Ausgestaltung sozialer Rechte und die Qualität der Lebensführung, die sie ermöglicht. Auf diese Weise lassen sich graduelle Abstufungen und wechselwirksam dimensionierte Aspekte von Soziallagen in den Blick nehmen und deren Rückwirkungen auf die Lebensführung ausloten. 
Regel für die Deckung der alltäglichen Lebenshaltungskosten aus; doch könnte etwa die Hälfte der Befragten einen gelegentlichen Urlaub, einen neuen Rechner oder gegebenenfalls eine Altersversorgung »ohne Mama im Rücken« nicht finanzieren. Stellt ersteres »nur« eine Einschränkung des Lebensstandards dar, ist die regelmäßige Anschaffung von Soft- und Hardware ihre Produktionsgrundlage, auf die sie nicht verzichten können. Und dass sich die meisten keine Altersvorsorge leisten können und ein Interviewter scheinbar amüsiert anmerkt, dass er lediglich eine Haftpflichtversicherung besitze, er sei halt Freiberufler, deutet die Härten ihrer sozialen Lage an. Die Befragten finden sich folglich in einer Situation wieder, die nichts mehr mit »schnellem Geld am Bildschirm « zu tun hat. Vielmehr sind sie nunmehr auf Marktnischen sowie auf ein potenziell dauerhaftes, niedriges Lohnniveau verwiesen.

So deuten die Befunde auf eine voranschreitende Prekarisierung ihrer Lebenslage hin, die sich auf folgende Punkte zuspitzen lässt (vgl. Dörre 2005). Erstens ist das Einkommen der Befragten unterdurchschnittlich, insbesondere im Verhältnis zu vergleichbaren Bildungsgruppen, und die eigenständige Existenzabsicherung ist nicht gewährleistet, zweitens ist der Zugang zu sozialen Teilhaberechten systematisch erschwert oder versperrt, die Abhängigkeit von privaten Netzen wie der Herkunftsfamilie steigt an, drittens wird eine souveräne Alltagsgestaltung und Lebensführung synchron sowie diachron erschwert und viertens werden durch das Zusammenwirken dieser Faktoren die Teilhabemöglichkeiten am gesellschaftlich üblichen - materiellen sowie immateriellen - Wohlstandsniveau beeinträchtigt.

\section{Marktstrategien}

Entlang der skizzierten Feldentwicklung werde ich nun darlegen, wie die Akteure das Spannungsfeld von marktgetriebener Bürokratisierung und Prekarisierungspotenzial nach dem Ende der »Dot-Com-Ära« gestalten.

Das Datenmaterial zeigt, dass in der Internetbranche drei Gruppen mit je spezifischen Profilen aufeinandertreffen und um die Vorherrschaft im Feld ringen. In Anlehnung an vertikale Beschreibungsmodelle habe ich eine Typologie entwickelt und sie Unternehmer, Dienstleister und Künstler genannt. Methodisch handelt es sich dabei um aus der Empirie gewonnene Realtypen. Ihre Profile verdeutlichen, auf welch unterschiedliche Weise die Anforderungen einer marktgetriebenen Bürokratisierung aufgegriffen werden. Zudem lässt sich nachzeichnen, dass Selbstbestimmung und soziale Deklassierung zwei Seiten derselben Medaille sind. Die subjektiv teils sehr ambivalenten Strategien zeigen ebenfalls, dass jene verbreitete Annahme, nach der hochqualifizierte Arbeitskraftunternehmer eine prekäre soziale Lage durch potenzielle Freiheitsgewinne subjektiv ausgleichen könnten, zu differenzieren ist. Schließlich macht die handlungsstrategische Schwankungsbreite der drei Typen Facetten des Zusammenhangs von radikal marktvermittelter und prekärer Lebenslage deutlich.

\section{$3.1 »$ Hauptsache aufwärts«: Die Unternehmer}

Als Unternehmer wurden fünf Befragte klassifiziert, drei Männer und zwei Frauen. Für sie ist die Internetbranche ein soziales und technisches Experimentierfeld. Sie sind Ende der 90er Jahre in die Branche eingestiegen und versprachen sich davon, »dass es da aufwärts geht « (060, männlich). Ihre Tätigkeit gehen sie als eine Art Nischenstrategie an, um sich Optionen für ihr wichtigstes Ziel zu eröffnen: die Unternehmer wollen es schaffen! Ihre Maxime heißt Selbstorganisation, Aufstieg und Leistung, kurz: »Es ist nicht wichtig, wie groß man ist, sondern wie groß man werden will« (060). Sie zeichnen sich durch einen Anlagesinn für eine profitable Verwertung ihrer Kompetenzen und Fähigkeiten aus. Ihr Einkommen ist vergleichsweise hoch. Sie zielen auf einen Statusgewinn, den sie durch ein spezifisch professionelles Arbeitshandeln umsetzen möchten. »Professionell« sein zieht sich als roter Faden durch diese Interviews und zeigt sich in nachfolgenden Dimensionen. 


\section{Modernisiert-traditionelle Arbeitsorganisation}

Die Unternehmer treten überwiegend direkt am Markt auf und agieren selten als Zulieferer für größere Projekte. Ihr Arbeitshandeln ist spezialisiert: Sie beschränken sich auf sogenannte Kernkompetenzen und am liebsten auf Managementtätigkeiten. Ihre Arbeitsabläufe versuchen sie weitgehend zu rationalisieren. Methodisches Arbeiten ist für sie der Inbegriff einer erfolgreichen Unternehmensführung. Spezialisiert sind die Unternehmer zudem auf »Industriekunden«, d.h. auf den finanziell vergleichsweise einträglichen Industrie- sowie produktionsorientierten Dienstleistungsbereich. Ihr Erfolgsrezept sei, dass sie arbeitsteilig produzierte »Komplettpakete« verkaufen:

Okay, wir sind 'ne Multimediaagentur, die sich auf einen bestimmten Bereich spezialisiert hat, nämlich auf Projektmanagment und -konzept-tion. Wir haben hier keine Produktion, wir machen keine Grafik, keinen E-Commerce, wir geben das alles raus. Wir sind sozusagen immer der administrative Kopf von so 'nem Projekt. (013, männlich)

$\mathrm{Zu}$ ihrem professionellen Selbstverständnis gehört auch eine informelle Verbetrieblichung. So vergleicht z.B. eine Interviewte die Zusammenarbeit mit ihrem Kollegen mit »Wie so zwei Anwälte in einer Sozietät; jeder für sich und trotzdem alle für eine Sache.« (017). Während sich jedoch Anwälte auf professionelle Berufsverbände verlassen können, handelt es sich hier um formale Alleindienstleister. Mittels einer nach ihren Maßstäben professionellen Corporate Identity wollen sie sich am Markt gut aufstellen, d.h. Synergieeffekte für den Marktzugang und -auftritt erzielen.

In ihren Ausführungen über optimale Arbeitsstrukturen spiegelt sich eine Mischung aus einem eher tayloristisch geprägten Arbeitsverständnis sowie einer vermarktlichten Kooperation wider. Zwar einerseits projektorientiert und auf externer Vermarktlichung beruhend (vgl. Sauer/Döhl 1997), verbinden die Unternehmer mit einer erfolgreichen Unternehmensund Personalführung relativ feste Arbeitszeiten und eindeutige Hierarchien. Zu ihrem Konzept des sozialen Aufstiegs gehört daher ebenso, dass sie sich als künftige Arbeitgeber sehen. Spätestens in fünf Jahren wollen sie sagen können, »so Jungs, jetzt Webdesign - macht Ihr mal.«(060) Derzeit sind sie jedoch auf andere Free Lancer angewiesen und halten diese für »unzuverlässig und unflexibel auch überraschenderweise« (013). Das Problem sei, dass jene kaum ergebnisorientiert arbeiten würden, sondern sich als »eigenwillige Künstler« (014) gerieren und nicht mit der nötigen Professionalität und Flexibilität agieren. Von (potenziellen) Mitarbeitern erwarten sie folgendes:

Aber denen musste dann auch sagen, pass mal auf, du wirst hier nicht bezahlt fürs Denken, du musst abarbeiten. Dann geht das. (017, weiblich)

Die Unternehmer zeichnen sich zudem durch eine ausgeprägte Kundenorientierung aus. Der »Endkunde« solle die Seiten nicht nur störungsfrei, sondern auch komfortabel nutzen können - dies sei ein Teil ihrer »Philosophie«. So stünden sie bei auftretenden Problemen »Gewehr bei Fuß«(017) und versprechen sich aus der Verbindung von einer ausgeprägten Kundenorientierung mit einer externen, projektorientierten Vermarktlichung Konkurrenzvorteile. Denn so seien nun mal die Spielregeln des Marktes, und denen müsse man gerecht werden.

\section{Formalisierte Konkurrenzbeziehungen}

Die Unternehmer organisieren ihre Konkurrenzbeziehungen vergleichsweise formell. Sie überlassen nichts dem Zufall und zunehmend auch weniger jenen vertrauensbasierten Netzwerken, die während der allgemeinen Internet-Euphorie tragfähig waren. So setzen sie auf KundInnennetzwerke und auf exklusive Lobbygruppen. Denn nur mit einem rundum methodischen Vorgehen, so ihre Einschätzung, lasse sich in diesem »komplett neuen Zweig Geld verdienen« (013). Die (vormaligen) Protagonisten der New Economy hätten demgegenüber 
unseriöse Geschäftsideen gehabt, sodass der Erfolg in Gestalt von Kunden und Verdienst ausblieb. »Wir waren ja auch nie New Economy« (017), denn die ist für sie der Inbegriff von Dilettantismus. Ein Befragter schildert seine Erfahrungen:

Die einen wollten Unterhosen, die anderen wollten Bücher [...] und ich hab's halt einfach nicht verstanden, wieso das funktionieren sollte [...] Es gab nie Anwendungen und offensichtlich gab's ja auch keine Kunden. Und das [...] war mir immer ein bisschen fremd. (013, männlich)

Verstand er sich gegen Ende der 90er Jahre als eine Randfigur des Geschehens, weil er sich bereits von Beginn an auf Industriekunden spezialisiert hatte und sich deshalb auf Networking Parties wie dem first oder last tuesday fremd fühlte, sieht er sich jetzt in seinem Vorgehen bestätigt. Er führt seinen Erfolg auf eben diese Spezialisierung zurück. Dennoch lassen sich die Unternehmer nicht den »Techie-Nerds« zuschlagen. Ihren Distinktionsgewinn ziehen sie vor allem aus ihrer funktionalen Spezialisierung sowie aus der Fokussierung auf »Industriekunden «, d.h. aus ihrem Anlagesinn, der sie in im kulturellen Sinne New-Economy fernen Marktsegmenten investieren ließ.

Zusammenfassend beschreiben die Unternehmer ihre Tätigkeit in der Internetbranche als einen Kampf, den sie als stimulierend sowie herausfordernd erleben und als notwendiges Element ihrer strategischen Ausrichtung erachten. Trotz aller Härten wie gesundheitliche Belastungen und bescheidende Verdienste haben sie sich bislang »für das Weiterkämpfen entschieden«, weil das »erfolgsorientierter sei« und man sich keinen Schikanen »von irgend einem Chef « aussetzen müsse, wie es eine Unternehmerin beschreibt. So drückt sich in ihren Äußerungen und Erzählungen ein idealtypisches Muster individualisierter Selbstverortung aus, das sie so zuspitzen:

Ich bin so selbständig, auf so einem hohen Level, dass ich das nur beschreiben kann als Unternehmer. (014, männlich)

Insgesamt agieren die Unternehmer vergleichsweise formalisiert und legen eine puritanische Arbeitsethik an den Tag. Sie greifen die Feldanforderungen offensiv auf und beschreiben auch die marktgetriebene Radikalisierung als positive Herausforderung, als marktbereinigenden Prozess, bei dem die »Spreu vom Weizen getrennt« worden sei. »Professionell« sein heißt für sie methodisches, rationalisiertes und »seriöses« (formalisiertes) Arbeiten, für das sie ihr Privatleben als temporäre Dispositionsmasse heran ziehen. Ihre Marktstrategie folgt einer funktionalen und rationalisierten Spezialisierung und offenbart eine recht traditionelle Aufstiegsorientierung.

\section{2 »Hauptsache sicher«: Die Dienstleister}

Als Dienstleister wurden elf Interviewte klassifiziert, sieben Frauen und vier Männer. Sie mündeten um das Jahr 2000 in die Branche ein. Fünf Fälle durchliefen eine vom Arbeitsamt finanzierte Weiterbildung. Sie repräsentieren daher einen halb-formalisierten Quereinstieg ins Feld.

Die Dienstleister zeichnet ein Sinn für das Machbare aus. Thema ihrer Fallgeschichten ist die Gratwanderung zwischen Sicherheit und Freiheit. Ihre Verdienstangaben streuen am stärksten und bewegen sich zwischen 960 und 1.920 Euro pro Monat. Aufstiegsorientierte Karriereambitionen oder Expansionspläne sind kein Bestandteil ihres Handlungshorizonts. Während die Unternehmer den Blick sozial eher nach oben richten, wenden Dienstleister den Blick eher nach unten und versuchen einen sozialen Abstieg abzuwehren, kurz: »Wonach ich mich am meisten sehne, ist so lukrativ zu arbeiten, dass man sich ein bisschen Sicherheit schafft.« (015, männlich) Der Sinn fürs Machbare zieht sich als roter Faden durch diese Interviews und zeigt sich in nachfolgenden Dimensionen. 


\section{Arbeitsorganisatorische Mischstrategie}

Die Dienstleister sind Allrounder und treten als auftragnehmende Agentur sowie als Direktanbieter auf. Sie steuern ihre Arbeit weniger methodisch als situativ. Sie sind in jeder Hinsicht vergleichsweise gering spezialisiert. Ihre Kunden stammen aus dem Dienstleistungsbereich jeglicher Art (Werbung, Unternehmensberatung, Kulturfestivals etc.). Trotz ihrer Vorliebe für den Kulturbereich nehmen die Dienstleister alle möglichen Aufträge an. »Ich sag mal Dienstleistung. Zwar ist es ein kreativer Prozess, aber letztlich eine Dienstleistung « (016), wie eine Befragte das Selbstverständnis in dieser Gruppe beschreibt.

Die geringe Spezialisierung auf ein spezifisches Marktsegment entspringt der Einschätzung, dass sie angesichts der Marktlage nicht wählerisch sein können. »Bodenständigkeit «, so ihre Erfahrung, ist die entscheidende »Überlebensgrundlage« (016). Denn nur so könnten sie dem Schicksal der New Economy entgehen. Dazu gehört etwa, dass sie »Freundschaftsdienste « ablehnen.

Du musst halt überleben. Und wenn du hier deine drei, vier Brötchen verdienen willst, dann musst du deine Zeit und Arbeit schon so kalkulieren, dass du anständig bezahlt wirst. (019, weiblich)

Demgegenüber ist die geringe Spezialisierung der Aufgaben- und Tätigkeitsprofile ihrer Auffassung über eine subjektiv befriedigende Erwerbsarbeit geschuldet. So ist ihr Arbeitsbegriff nach einer formalen und inhaltlichen Ebene kategorisiert. Sie differenzieren zwischen gebrauchswertorientierter und tauschwertorientierter Arbeit und folgen insofern einer arbeitsorganisatorischen Mischstrategie. Eine Interviewte erklärt, wie sie das macht:

(E)s gibt natürlich Kunden, z.B. für die eine Freundin, die hat so eine Videoschnittfirma, und für die die Seite zu machen, das macht Spaß [...] Solche Sachen, da verdient man halt nix, aber [...] da kann ich hinter stehen. Aber jetzt machen wir grad was für eine Immobilienfirma. Das werden tolle Seiten, die sehen grafisch wirklich gut aus, aber inhaltlich? Ja, ist nicht so. (040)

Neben der Absicherungsmentalität sind die Dienstleister dadurch charakterisiert, dass sie »ein nettes Leben« führen möchten. Dafür schrauben sie etwaige Karrierevorstellungen und finanziellen Erfolg zurück.

(D)ass ich jetzt nicht sage, ich will auf Teufel komm raus Karriere machen [...] Das ist irgendwie nicht mein Ziel, [...] sondern ich will auch das Gefühl haben, ein nettes Leben zu führen. (015, männlich)

Das Gefühl und die Lust an der Arbeit ist für die Dienstleister neben der »Nähe zu (ihren) Zahlen« (016) ein wichtiges Moment des Arbeitsalltags. Das heißt aber nicht, dass sie nur arbeiten, wenn sie Lust dazu haben. Sie begreifen ihre Arbeit als einen kreativen Prozess, der seinen eigenen Rhythmus habe, »weil wenn ich gerade die Idee habe, dann muss ich sie gleich festhalten, sonst ist sie weg.«(016) Infolge dieser vergleichsweise irrationalen Arbeitshaltung greift Arbeit gewissermaßen auf sie zu; egal zu welcher Tages- oder Nachtzeit. Dennoch scheinen sich die Dienstleister Freiräume auch während gängiger »Kernarbeitszeiten « offen halten zu können. Ein wichtiger Grad von selbst bestimmter Arbeit ist für sie folglich, dass sie ihre Arbeit nicht methodisch planen und sich keinem festen Rhythmus unterwerfen. Dazu gehört auch ein flexibler Umgang mit ihren Arbeitszeiten.

\section{Informelle Konkurrenzbeziehungen}

Dienstleister verlassen sich bei der Auftragssuche überwiegend auf persönliche Kontakte. Nur scheinbar im Gegensatz zum Ziel der Statussicherung steht, dass sie die Konkurrenz und dem damit verknüpften Kampf um soziale Stellungen vergleichsweise oft dem Zufall überlassen: Sie verfahren hier nach dem Motto »Hey, brauchst du nicht ...«. Allerdings kennzeichnen sie diese laxe Haltung als einen Konflikt, der sich aus dem Spannungsverhältnis von Existenz absichern vs. ein nettes Leben haben zu wollen, einstellt. So seien sie für eine dezidierte Kunden- und Akquisearbeit nicht »so der Typ für« (015), bewundern jedoch all je- 
ne, die das »Klinkenputzen« (015) beherrschen würden. Doch so lange sie sich über Wasser halten können, leisten sie sich den Luxus informellen und eher vertrauensbasierten Konkurrenzverhaltens. Hinzu kommt eine kundenorientierte Preispolitik, die sie als »Robin-HoodManier« (016) bezeichnen, weil sie von potenziell zahlungskräftigen Kunden vergleichsweise hohe Honorare verlangen. Als Bestandteil ihrer Mischstrategie erlaubt diese Praxis, auch weniger einträgliche Projekte aus dem Kulturbereich zu bearbeiten wie z.B. den Internetauftritt eines Off-Filmfestivals.

Dennoch erleben es die Dienstleister vielfach als beängstigend, »dass ich tatsächlich irgend so was wie ein kleines Unternehmen bin« (070). So ist ihre Lust an der Arbeit häufig von Existenzängsten überschattet, »weil das einfach so unsicher ist «(020). Dennoch sind sie bereit, vergleichsweise gering entlohnte Tätigkeiten auszuführen, sofern sie sich davon einen individuellen Mehrwert versprechen; wobei sich Mehrwert für sie primär in kreativer Entfaltung bemisst.

Das besondere Kennzeichen der Dienstleister ist also erstens, dass sie Existenzängste mitunter drastisch thematisieren. Zweitens stellt die Internetbranche für sie ein vergleichsweise »normales « Erwerbsfeld dar, das ihnen gleichwohl höhere Subjektivierungsoptionen eröffnet, als sie sich von konventionellen Erwerbsfeldern erwarten. Drittens ist Erwerbsarbeit für sie nur das halbe Leben; sie sind nicht bereit, ihre Lebensführung vollständig auf erwerbsbezogene Anforderungen auszurichten. Ihre Marktstrategie ist ein Kompromiss zwischen marktbezogenen Anforderungen und »lebensweltlichen« Bedürfnissen und zielt auf eine Statussicherung.

\section{3 »Hauptsache unabhängig«: Die Künstler}

Als Künstler wurden drei Interviewte klassifiziert, zwei Männer und eine Frau. Sie sind buchstäbliche Künstler und haben einen kunstbezogenen Bildungshintergrund - in zwei der drei Fälle allerdings ohne Studienabschluss. Sie waren bereits als Webdesigner tätig, als sich für die Bundesrepublik noch längst nicht von einer Internetbranche sprechen ließ. Sie streben inhaltliche Perfektion und Selbstverwirklichung, aber nicht unbedingt Professionalität an. Ihr herausragendes Charakteristikum ist, dass sie die gleichen Dinge anders machen wollen. Die Internetbranche betrachten sie als künstlerisches Produktionsfeld mit intendierten Verdiensteffekten, weil »es geht nicht um Arbeit oder was man sich darunter, also, wenn du so willst, ist ein bisschen Bohèmeleben auch.« (021, männlich). Ihr Verdienst ist der geringste im Sample.

Ihre Fallgeschichten erzählen am deutlichsten vom Kampf um Vorherrschaft und Verdrängung in der Internetbranche. Als Pioniere bereiteten sie das Feld für Nachkömmlinge auf und sind nun ihrerseits von Verdrängung bedroht (vgl. Blasius/Dangschat 1990). Ihr treten sie mit symbolischen Distinktionsstrategien entgegen, um sich als legitime Feldzugehörige auszuweisen.

\section{Arbeitsinhaltliche Spezialisierung}

Ihr Arbeitshandeln stellt gängige Vorstellungen von Arbeit am stärksten in Frage. Ihre Tätigkeit begreifen sie als »digitales Kunsthandwerk « (021). Ihnen geht es darum, sich von der »sklavischen Notdurft« des Arbeitens zu befreien, um sich Freiräume für ihre eigentliche Tätigkeit zu schaffen. Deutlicher als die Dienstleister unterscheiden die Künstler zwischen Herstellen und Arbeiten. Die Künstler sind spezialisiert, jedoch vor allem auf das künstlerische Marktsegment.

Für dieses »Bohèmeleben« werden finanzielle Engpässe in Kauf genommen. Es scheint so, dass ökonomisches Kapital weitgehend durch inhaltliche Selbstbestimmung ersetzt wird. 
So heben sie hervor, dass sie den Gürtel lieber enger schnallen, als den alltäglichen Kampf um Aufträge mitzumachen und sinnlose Seiten wie z.B. Konsumwerbung zu gestalten. Die Künstlerin bringt es am deutlichsten auf den Punkt: »Ich möchte nicht an Martinis Kampagne arbeiten. Also, das ist mir ehrlich gesagt ein bisschen zu hohl.«(018) Gleichwohl räumt ein anderer ein, dass diese Schwerpunktsetzung auf der einen Seite »zwar schön (sei), weil die Projekte interessant sind, aber finanziell ist das natürlich nicht so gesund auf Dauer.« (080)

\section{Informelle Konkurrenzbeziehungen}

Auch die Künstler konstatieren, dass die Konkurrenz in den vergangenen Jahren härter geworden sei. Dennoch verlassen sie sich, wie die Dienstleister, eher auf persönliche Kontakte. Parallelen zeigen sich hier beispielsweise in einer ambivalenten Haltung zu ihren Marktbehauptungsstrategien. Eines ihrer Grundprobleme sei, dass sie zu viel dem Zufall überlassen. So sind sich die Künstler der Markt- und Existenzzwänge sehr wohl bewusst - und nehmen ebenfalls, was kommt. Doch aufgrund ihrer Spezialisierung auf den Kunstmarkt »kommt« nur in Ausnahmefällen und auf Empfehlung ein vergleichsweise lukrativer Auftrag herein. Und bislang hat niemand von ihnen ein Projekt aus dem produktionsorientierten Dienstleistungsbereich akquirieren können und/oder wollen.

Derweil ein informeller Kampf bei den Dienstleistern als ein Aspekt ihrer Mischstrategie identifiziert wurde, ist der entscheidende Handlungsmotor der Künstler, »den materiellen Sinn dem anderen anzugleichen.«(080) In Folge der Kommerzialisierung strömten nun Menschen in das Feld, die keinerlei inhaltliche Bindung mehr ans Medium haben, sondern es nur mehr als Einkommensquelle oder noch schlimmer: Webdesign als second best choice betrachten.

Also, ich meine, jeder kann jetzt mittlerweile Webdesign [...] als ich damit angefangen habe, konnte das nicht jeder und dann sind die Arbeitslosen zur [...] Webdesignumschulung gegangen. Und meinst $\mathrm{du}$, die machen was damit? (018, weiblich)

Imageprojekte, um in der gegenwärtigen Marktlage Fuß zu fassen, lehnen sie ab und überlassen es lieber anderen, »irgendwelchen Scheiß für irgend einen Werbeträger « zu machen: »Also das ist bah!«(018) Explizit machen sie diese Haltung, wenn sie etwa vom Mut zum Dilettantismus sprechen. Während sich die Unternehmer gegen jedwede Form des »Dilettantismus « abgrenzen und »Professionalität« anstreben, heben ihn die Künstler positiv als Improvisationstalent und Flexibilität hervor. »Leistung « und »Existenzsicherung « hat für sie daher auf den ersten Blick wenig mit »Markterfolg « zu tun. So drückt der Mut zum Dilettantismus die Abneigung gegen Selbst-Vermarktungszwänge aus.

Doch zeigt sich auf den zweiten Blick, dass »Selbstverwirklichung « nicht ohne »Markterfolg « zu verstehen ist. Denn für ihre konsequente Strategie sich abseits von Marktzwängen sozial zu verorten, bezahlen sie den Preis des geringsten Verdienstes im Sample. Zugleich ist das Bestreben die gleichen Dinge anders zu machen die vergleichsweise konsequenteste Strategie für eine Einpassung ihres Arbeitshandelns in eine subjektiv befriedigende Erwerbsarbeit. Doch erfordert ihr Arbeitsverständnis offenbar eine bedingungslose Hingabe. So zeigt sich, dass die Künstler ähnlich zeitintensiv arbeiten wie die Unternehmer, wenn auch auf Basis gänzlich anderer Motive. Die Künstler versuchen sich im Feld durch eine Spezialisierung auf den Kunstmarkt und mittels symbolischer Distinktionsstrategien, dem Rückzug auf den Nominalwert ihrer Arbeitsleistung, zu halten. Sie bezahlen dafür den Preis einer randständigen Marktlage und des vergleichsweise geringsten Verdienstes. 


\section{Fazit: Prekarisierung auf hohem Niveau}

Der rapide Strukturwandel der Internetbranche und seine Folgen für die soziale Lage von Webdesignern geben Aufschluss über Aspekte des Strukturwandels von Arbeit, die sich bislang zwar kaum mittels aggregierter Daten nachweisen lassen - die aber nichtsdestoweniger auf einen »sozialstrukturellen Zwischenraum « (Castel 2000) verweisen, in dem eine Privatisierung sozialer Risiken und gesellschaftlicher Kosten stattfindet.

So ist es einerseits richtig, auf die Abspaltung sozialer und materieller Randlagen und damit auf persistente Problemlagen hinzuweisen (etwa Groh-Samberg 2005) und den Begriff der Exklusion in letzter Konsequenz für die Zone der Entkopplung am Rand der Gesellschaft zu reservieren (Castel 2000). Dennoch dürfen andererseits diese Prozesse den Blick nicht »für die allmähliche Erosion und Gefährdung sozialer Stabilität, für die Prekarität des Wohlstands, für die Verwundbarkeit beruflicher Positionen und gesellschaftlicher Beziehungen « verstellen (Vogel 2004, S. 41). Denn was in den Debatten noch wenig beachtet wird, sind die Grauzonen sozialstrukturellen Wandels, in denen es um Abstiegs- und Deklassierungsdrohungen, um Aufstiegs- und Stabilitätshoffnungen, aber eben nicht um Exklusionsgewissheiten geht. Die Befunde zeigen, dass sich die Untersuchungsgruppe in einem solch sozialstrukturellen Zwischenraum befindet.

Fassen wir also zusammen: Die handlungsstrategischen Praxen der als Unternehmer, Dienstleister und Künstler klassifizierten Interviewten sind Stellungnahmen innerhalb der Internetbranche nach dem Hype. Sie erzählen von feldspezifischen Entwicklungen und davon, wie sich die Interviewten mit den veränderten Feldbedingungen praktisch auseinandersetzen und sich soziale Verfügungsmacht im Feld erschließen. War ihre unmittelbare Marktabhängigkeit zudem während der Expansionsphase der Internetbranche bedingt problematisch, spitzt sie sich nach 2001 zu und lässt feldinterne, ungleichheitsgenerierende Effekte und deutliche Positionskämpfe um die Bedingungen legitimer Teilhabe am Feld erkennen. Soziales Kapital sowie netzwerkgetragene Konkurrenzstrukturen stellen dabei eine entscheidende Ressource auf dem Marktplatz Internetbranche dar und stiften zugleich lediglich partikulare Integration. Teilhabe ist in diesem Fall folglich die Kehrseite einer gesellschaftlichen Ausgrenzungslage.

Die Untersuchungsbefunde zeigen, dass jene, die nur über Freunde und Kollegen akquirieren zum einen vergleichsweise kurz im Geschäft sind, zum zweiten nur wenig spezialisiert sind und zum dritten vorwiegend milieunahe Auftraggeber haben. Zudem erzielen jene, die sich auf die Erstellung kunst- und kulturbezogener Websites kaprizieren, vergleichsweise geringe Honorare.

Demgegenüber erzielen jene Befragten hohe Honorare und eine hohe Auftragsdichte, kurz Konkurrenzvorteile, welche die neuen Anforderungen vergleichsweise offensiv aufgreifen. So spezialisieren sich vornehmlich die Unternehmer auf finanziell lukrative Marktsegmente, bieten arbeitsteilig erstellte »Komplettpakete« an und formalisieren ihr Konkurrenzverhalten, indem sie sich auf Lobbygruppen sowie auf vertikale Kundenkontakte fokussieren, antworten also auf den steigenden Konkurrenzkampf mit verschiedenen Rationalisierungstechniken. Anders die Künstler. Sie haben die größten Probleme, sich im Feld zu halten. Denn ihre Marktstrategie belegt, dass sie die Internetbranche erst sekundär als einen Erwerbsmarkt betrachten. Primär stellt diese für sie ein künstlerisches Produktionsfeld mit intendierten Verdiensteffekten dar. Die große Anzahl der Dienstleister nährt zudem die Hypothese, dass die wachsende Formalisierung des Feldes einem sozialen Schließungsprozess entspricht, der Free Lancer auf Branchennischen sowie auf ein nachhaltig niedriges Lohnniveau und auf eine dauerhaft fragile Existenz verweist. 
Während sich also die Befragten über ihre Marktstrategien jeweils unterschiedliche Teilhabeoptionen in der Internetbranche erschließen, sind diese andererseits im Zusammenhang mit einer Soziallage zu sehen, die sich generell in einem sozialen Zwischenraum von Privilegierung und Prekarisierung befindet. Zwar besitzen Webdesigner aufgrund ihres hohen Bildungsniveaus wichtige Ressourcen, um berufliche und soziale Instabilitäten zu bewältigen; dennoch ist ihre soziale Lage äußerst verwundbar. So verengt sich der angesprochene Zwischenraum im Verlauf der Feldkontraktion zusehends und strukturiert sich entlang der jeweiligen Marktstrategien. Vor dem Hintergrund der konstatierten marktgetriebenen Bürokratisierung wird er zu einer typologisch differenzierten Prekarisierung auf hohem Niveau deren handlungsstrategische Differenzierung die Bandbreite von prekären Lebenslagen andeutet.

Dass diese Soziallage von den Befragten jeweils unterschiedlich als selbstbestimmt erlebt wird, hat sicherlich wenig damit zu tun, dass sie sich das eigene Elend schön reden. Vielmehr verweisen die empirischen Befunde einerseits darauf, welch hohe Ressourcenausstattung es braucht, um sich in sozialstrukturellen Zwischenräumen zurecht zu finden, deren Ungewissheiten zu erkennen und zu gestalten.

Andererseits sprechen die Befunde dafür, dass sich Ungewissheiten und Entsicherungen durchaus in die soziale Mitte ausdehnen; und zwar nicht nur als gefühlte Unsicherheiten, sondern als praktisch erfahrene, welche die Akteure relativ nüchtern als »neue Normalität « deuten.

\section{Literatur}

Batt, Rosemary et.al. (2001): NetWorking. Work Patterns and the Workforce Policies for the New Media Industry, Economic Policy Institute. Washington D.C.

Beckert, Jens (1997): Grenzen des Marktes. Die sozialen Grundlagen wirtschaftlicher Effizienz, Frankfurt, M.-New York.

Blasius, Jörg/Dangschat, Jens (Hrsg.) (1990): Gentrification: Die Aufwertung innenstadtnaher Wohnviertel, Frankfurt/M.-New York.

Bögenhold, Dieter/Leicht, René (2000): Neue Selbständigkeit und Entrepreneurship: Moderne Vokabeln und damit verbundene Hoffnungen und Irrtümer, in: WSI-Mitteilungen 53, S. 779-787.

Bourdieu, Pierre (1983): Ökonomisches Kapital, kulturelles Kapital, soziales Kapital, in: R. Kreckel (Hrsg.): Soziale Ungleichheiten; Soziale Welt, Sonderband 2, S. 183-198.

Bourdieu, Pierre (1987): Die feinen Unterschiede. Kritik der gesellschaftlichen Urteilskraft, Frankfurt/M.

Bourdieu, Pierre (1997): Das literarische Feld, in: P. Bourdieu/L. Pinto (Hrsg.) Streifzüge durch das literarische Feld, Konstanz, S. 33-147.

Bourdieu, Pierre et al. (2005): Das Elend der Welt. Studienausgabe, Stuttgart.

Castel, Robert (2000): Die Metamorphosen der sozialen Frage. Eine Chronik der Lohnarbeit, Konstanz.

Castells, Manuel (2001): Das Informationszeitalter I: Die Netzwerkgesellschaft, Opladen.

Christopherson, Susan (2001): Does Risk Require Trust?, in: Environment and Planning: A. Manuskript.

Dörre, Klaus (2005): Prekarität - eine arbeitspolitische Herausforderung, in: WSI-Mitteilungen 58, S. 250-258.

Gill, Rosalind (2002): Cool, Creative and Egalitarian? Exploring Gender in Project-Based New Media Work in Europe, in: Information, Communication \& Society 5, S. 70-89.

Gottschall, Karin/Schnell, Christiane (2000): Alleindienstleister in Kulturberufen - Zwischen neuer Selbständigkeit und alten Abhängigkeiten, in: WSI-Mitteilungen 53, S. 804-810.

Granovetter, Mark (1973): The Strength of Weak Ties, in: American Journal of Sociology 78, S. 13601380.

Granovetter, Mark (1985): Economic action and social structure. The problem of embeddedness, in: American Journal of Sociology 91, S. 481-510. 
Groh-Samberg, Olaf (2005): Die Aktualität der sozialen Frage - Trendanalysen sozialer Ausgrenzung 1984-2004, in: WSI-Mitteilungen 11, S. 616-623.

Haak, Carol/Schmid, Günter (2001): Arbeitsmärkte für Künstler und Publizisten: Modelle der künftigen Arbeitswelt? in: Leviathan. Zeitschrift für Sozialwissenschaft 29, S. 156-178.

Henninger, Annette (2004): Free Lancer in den Neuen Medien: Jenseits standardisierter Muster von Arbeit und Leben? in: H. Kahlert/C. Kajatin (Hrsg.): Arbeit und Vernetzung im Informationszeitalter. Wie neue Technologien die Geschlechterverhältnisse verändern, Frankfurt, M.-New York, S. 143-166.

Honneth, Axel (Hrsg.) (2002): Befreiung aus der Mündigkeit. Paradoxien des gegenwärtigen Kapitalismus, Frankfurt/M.-New York.

Jansen, Dorothea (2003): Einführung in die Netzwerkanalyse, Opladen.

Kaufmann, Franz Xaver (2003): Sozialpolitisches Denken, Frankfurt/M.

Kotamraju, Nalini P. (2002): Keeping Up: Web Design Skill and the Reinvented Worker, in: Information, Communication \& Society 5, S. 1-26.

Kraemer, Klaus (1997): Der Markt der Gesellschaft, Frankfurt, M.-New York.

Leicht, René; Philipp, Ralf (2005): Die wachsende Bedeutung von Ein-Personen-Unternehmen in Deutschland: Wo und unter welchen Bedingungen arbeiten Selbständige zunehmend alleine? in: F. Welter (Hrsg.): Dynamik im Unternehmenssektor: Theorie, Empirie und Politik, Berlin, S. 131-154.

Manske, Alexandra (2005): Zum ungleichen Wert von Sozialkapital. Netzwerke aus einer Perspektive sozialer Praxis. Vortragsmanuskript, Sektionstagung Soziale Ungleichheit und Sozialstrukturanalyse der DGS, Bielefeld 11`05.

Marshall, Thomas H. (1992): Bürgerrechte und soziale Klassen. Zur Soziologie des Wohlfahrtsstaats. Frankfurt/M.-New York.

Mayer-Ahuja, Nicole/Wolf, Harald (2004): Jenseits des Hype: Arbeit bei Internetdienstleistern, in: SOFIMitteilungen 32, S. 79-96.

Meschnig, Alexander/Stuhr, Matthias (2001): www.revolution.de. Die Kultur der New Economy, Hamburg.

Müller, Walter (1998): Erwartete und unerwartete Folgen der Bildungsexpansion, in: J. Friedrichs/R.M. Lepsius/K.U. Mayer (Hrsg.): Die Diagnosefähigkeit der Soziologie. Kölner Zeitschrift für Soziologie und Sozialpsychologie, Sonderheft, S. 81-112.

Neckel, Sighard (2001): »Leistung « und »Erfolg«. Die symbolische Ordnung der Marktgesellschaft, in: E. Barlösius/ H.-P. Müller/ S. Sigmund (Hrsg.): Gesellschaftsbilder im Umbruch. Soziologische Perspektiven in Deutschland, Opladen, S. 245-268.

Neckel, Sighard/Dröge, Kai/Somm, Irene (2005): Das umkämpfte Leistungsprinzip - Deutungskonflikte um die Legitimationen sozialer Ungleichheit, in: WSI-Mitteilungen 58, S. 368-374.

Neff, Gina (2002): Game Over. With the collapse of the new economy complete, yesterday's dot-comers are only now beginning to understand the odds, in: The American Prospect 13, S. 15-17.

Neff, Gina/Wissinger, Elizabeth/Zukin, Sharon (2001): »Cool« Jobs in »Hot” Industries. Fahion Models and New Media Workers as Entrepreneurial Labor. Manuskript.

Nullmeier, Frank (2001): Was folgt auf den »Sieg des Marktes«? In: E. Barlösius/H.-P. Müller/S. Sigmund (Hrsg.): Gesellschaftsbilder im Umbruch. Soziologische Perspektiven in Deutschland, Opladen, S. 227-244.

Ortlieb, Renate/Rokitte, Simone (2004): New Economy - neue Geschlechterverhältnisse? In: U. Pasero/ B.P. Priddat (Hrsg.): Organisationen und Netzwerke: Der Fall Gender. Wiesbaden, S. 113-142.

Perrons, Diane (2003): The New Economy and the Work-Life-Balance: Conceptual Explorations and a Case Study of New Media, in: Gender, Work and Organization 10, S. 65-93.

Pratt, Andy C. (2000): New media, the new economy, and new spaces, in: Geoforum 31, S. 25-436.

Rehberg, Frank/Stöger, Ursula/Sträter, Detlev (2002): Frauen in der Medienwirtschaft: Chancen und Hemmnisse für Frauenerwerbstätigkeit in einer prosperierenden Zukunftsbranche, München.

Rüb, Friedbert (2003): Vom Wohlfahrtsstaat zum »manageriellen Staat «? Zum Wandel des Verhältnisses von Markt und Staat in der deutschen Sozialpolitik, in: R. Czada/R. Zintl (Hrsg.): Politik und Markt. PVS-Politische Vierteljahreszeitschrift. Sonderheft 34, S. 256-300. 
Sauer, Dieter/Döhl, Volker (1997): Die Auflösung des Unternehmens? - Entwicklungstendenzen der Unternehmensreorganisation in den 90er Jahren, in: ISF-München (Hrsg.): Jahrbuch Sozialwissenschaftliche Technikberichterstattung 1996, Berlin, S. 19-76.

Simmel, Georg (1983): Soziologie der Konkurrenz. In: H. Dahme/O. Rammstedt (Hrsg.): Schriften zur Soziologie. Eine Auswahl, Frankfurt/M., S. 173-193.

Strauss, Anselm (1998): Grundlage qualitativer Sozialforschung. Datenanalyse und Theoriebildung, München.

Stuhr, Mathias (2003): Popökonomie. Eine Reformation zwischen Lifestyle und Gegenkultur, in: A. Meschnig/M. Stuhr (Hrsg.): Arbeit als Lebensstil, Frankfurt/M., S. 162-184.

Swedberg, Richard (1994): Markets as Social Structures, in: P. Smelser/R. Swedberg (Hrsg): The Handbook of Economic Sociology, New York, S. 255-282.

Vanselow, Achim (2002): Neue Selbständigkeit in der New Economy - Beobachtungen zur Situation von »E-Lancern« in Deutschland, in: H. Eichmann/I. Kaupa/K. Steiner (Hrsg.): Game Over? Neue Selbstständigkeit und New Economy nach dem Hype. Wien, S. 157-184.

Vester, Michael et al. (2001): Soziale Milieus im gesellschaftlichen Strukturwandel. Zwischen Integration und Ausgrenzung, Frankfurt/M.

Vogel, Berthold (2004): Der Nachmittag des Wohlfahrtsstaats. Zur politischen Ordnung gesellschaftlicher Ungleichheit, in: Mittelweg 36 13, S. 36-56.

Voswinkel, Stephan (2000): Transformation des Marktes in marktorientierten Organisationen. In: H.-G. Brose, (Hrsg.): Die Reorganisation der Arbeitsgesellschaft. Frankfurt,M.-New York, S. 239-274.

Weber, Max (1972): Wirtschaft und Gesellschaft. Grundriss der verstehenden Soziologie. 5. revidierte Ausgabe, besorgt von J. Winckelmann, Tübingen.

Wolf, Harald (2004): Institutionalisierung von Herrschaft in neuen Dienstleistungsfeldern - das Beispiel der Internetbranche. Beitrag zur Adhoc-Gruppe »Dienstleistungsarbeit und soziale Ungleichheit - Herausforderungen für die Industriesoziologie« auf dem 32. Kongress der DGS, 10`04, München, Manuskript.

Alexandra Manske, Institut für Gesellschaftswissenschaften, FG Politikwissenschaft, TU Berlin, Franklinstr. 28-29, 10587 Berlin alexandra.manske@tu-berlin.de 